Toxicology $128(1998) 181-189$

\title{
Antioxidant effects of $N$-acetylcysteine and succimer in red blood cells from lead-exposed rats
}

\author{
Hande Gürer ${ }^{\text {a }}$, Hilal Özgünes ${ }^{\text {b }}$, Rachel Neal ${ }^{\text {a }}$, Douglas R. Spitz ${ }^{\mathrm{c}}$, Nuran Erçal ${ }^{\mathrm{a}, *}$ \\ a Department of Chemistry, University of Missouri-Rolla, 142 Schrenk Hall, Rolla, MO 65409, USA \\ ${ }^{\mathrm{b}}$ Department of Toxicology, University of Hacettepe, Ankara, Turkey \\ ${ }^{\mathrm{c}}$ Section of Cancer Biology, Radiation Oncology Center, Washington University School of Medicine, St. Louis MO, USA
}

Received 28 January 1998; accepted 19 May 1998

\begin{abstract}
This study examined whether lead-induced alterations in selected parameters that are indicative of oxidative stress accompany the toxic effects of lead in red blood cells (RBCs) in vivo. It also explored the possibility that treatment with $\mathrm{N}$-acetylcysteine (NAC) or succimer (meso-2,3-dimercaptosuccinic acid) was capable of reversing parameters indicative of lead-induced oxidative stress. Fisher 344 rats were given 2000 ppm lead acetate in their drinking water for 5 weeks. The lead was then removed and the animals were given NAC $(800 \mathrm{mg} / \mathrm{kg} / \mathrm{day})$ or succimer $(90$ $\mathrm{mg} / \mathrm{kg} /$ day) in their drinking water for 1 week, after which the RBCs were harvested. Animals not given lead and those given lead, but not NAC or succimer, served as negative and positive controls, respectively. At the end of the experiment, blood-lead levels were $35 \pm 4 \mu \mathrm{g} / \mathrm{dl}$ in lead-treated animals, which were reduced to $2.5 \pm 1 \mu \mathrm{g} / \mathrm{dl}$ by treatment with succimer and to $25 \pm 3 \mu \mathrm{g} / \mathrm{dl}$ by treatment with NAC. Lead-exposed animals demonstrated signs of anemia as evidenced by anisocytosis, poikilocytosis, and alterations in hemoglobin, hematocrit, and mean corpuscular volume. Lipid peroxidation, as evidenced by increased malondialdehyde (MDA) content, as well as decreases in reduced glutathione (GSH) and increases in catalase and glucose 6-phosphate dehydrogenase (G6PD) activity were noted in RBCs from lead-treated rats, suggesting that the lead induced oxidative stress. In addition, a significant reduction in blood $\delta$-aminolevulinic acid dehydratase (ALAD) activity suggested that accumulation and autooxidation of $\delta$-aminolevulinic acid might contribute to lead-induced oxidative stress. Treatment with either NAC or succimer reversed lead-induced alterations in MDA and GSH content, but only succimer appeared to partially restore ALAD activity. These results provide in vivo evidence supporting the hypothesis that lead induces oxidative stress in RBCs, which is reversible by treatment with a thiol antioxidant (NAC), as well as a chelating agent (succimer). (C) 1998 Elsevier Science Ireland Ltd. All rights reserved.
\end{abstract}

Keywords: Lead; Oxidative stress; NAC; Succimer; Red blood cells

*Corresponding author. Tel.: + 1573 3416950; fax: + 1573 3416033; e-mail: nercal@umr.edu 


\section{Introduction}

Lead is a toxic heavy metal which induces a broad range of physiological, biochemical, and behavioral dysfunctions. One of the targets for lead toxicity is the hematological system. It is well established that lead induces changes in the composition of red blood cell (RBC) membrane proteins (Fukumoto et al., 1983), reduces the membrane $\mathrm{Na}^{+} / \mathrm{K}^{+}$ATPase activity (Hasan et al., 1971), and inhibits hemoglobin synthesis (Waldron, 1966; Haeger-Aronsen et al., 1971; Ratcliffe, 1981). However, the mechanism(s) responsible for the toxic effects of lead on RBCs (Waldron, 1966; Ratcliffe, 1981; Sugawara et al., 1991) are not altogether clear.

Previous animal studies (Lawton and Donaldson, 1991; Sandhir et al., 1994; Sandhir and Gill, 1995), including our own (Ercal et al., 1996), have suggested that lead is capable of causing oxidative stress in the liver, kidney, and brain. In vitro studies have suggested that lead also induces oxidative stress in RBCs (Ribarov and Benov, 1981; Ribarov et al., 1981; Quinlan et al., 1988). This hypothesis was further supported by studies where the oxidative damage was demonstrated in RBCs of lead-exposed workers (Ito et al., 1985; Monteiro et al., 1985; Sugawara et al., 1991). The potential role for oxidative stress in injury, associated with lead poisoning, suggests that antioxidants may enhance the efficacy of treatment protocols designed to mitigate lead-induced toxicity.

$\mathrm{N}$-acetylcysteine (NAC) is a thiol-containing antioxidant (Fig. 1) which has been utilized to mitigate various conditions of oxidative stress. Its antioxidant action is believed to originate from its ability to stimulate glutathione (GSH) synthesis, therefore maintaining intracellular GSH levels (Moldeus et al., 1986) and scavenging reactive oxygen species (ROS) (Aruoma et al., 1989). Previous studies also indicated that NAC has chelating activity against some heavy metals such as boron and chromium (Banner et al., 1986). NAC has been used in several clinical conditions over the years with few adverse side effects (Prescott et al., 1977; Ziment, 1986). All of these beneficial properties led us to consider NAC as a strong candidate for restoring impaired prooxidant/antioxidant balance in lead poisoning.

Succimer (meso-2,3-dimercaptosuccinic acid) is an oral chelating agent that was approved by the U.S. Food and Drug Administration in 1991 for the treatment of lead poisoning in children. Although it has been shown to have several advantages, compared to conventional chelating agents, its usage is limited because of the lack of clinical data (Center for Disease Control, 1991; Aposhian et al., 1995). Two sulfhydryl groups in the structure of the compound suggest that, in addition to chelation, the antioxidant activity of succimer may be useful in the treatment of lead poisoning (Fig. 1).

The present studies investigate whether lead-induced oxidative stress accompanies lead-induced injury to RBCs from Fisher 344 rats that were fed lead acetate in their drinking water. In addition, the ability of NAC or succimer to reverse parameters of oxidative stress and injury following lead exposure was evaluated in RBCs from lead-exposed animals. Since succimer is a well-known chelator and NAC can potentially function as a chelating agent, blood lead levels were determined in all treatment groups. Selected parameters indicative of oxidative stress were evaluated in RBCs from all treatment groups. Malondialde-
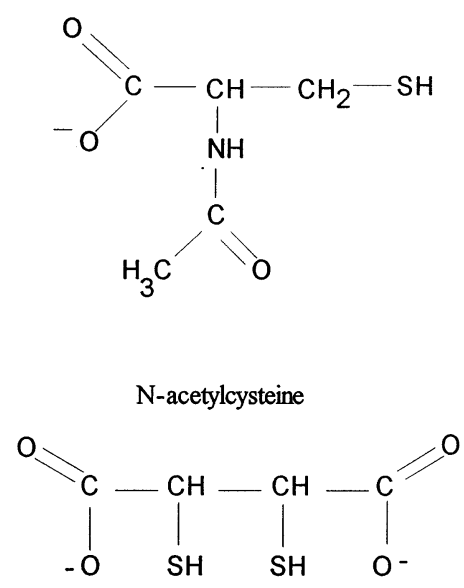

Succimer (meso-2,3-dimercaptosuccinic acid)

Fig. 1. Chemical structures of $N$-acetylcysteine and succimer. 
hyde (MDA) content was evaluated as an endpoint indicative of the extent of lipid peroxidation, since MDA is known to be one of the most abundant aldehydes formed as a byproduct of lipid peroxidation. Reduced glutathione (GSH) content was determined as a measure of intracellular thiol antioxidant status and glucose 6-phosphate dehydrogenase (G6PD), as well as catalase (CAT) activity, were evaluated as key components of cellular defenses against hydroperoxides. Blood $\delta$-aminolevulinic acid dehydratase (ALAD) activity was also determined, since lead-induced inhibition of this enzyme has been suggested to result in the accumulation of $\delta$-aminolevulinic acid, and its autooxidation has been shown to generate ROS such as superoxide and hydrogen peroxide (Monteiro et al., 1986). The results of this study indicate that, (1) lead is capable of causing increases in parameters that are indicative of oxidative stress in RBCs following lead exposure, and (2) treatment with NAC or succimer is capable of reversing these parameters.

\section{Materials and methods}

The $\quad N$-(1-pyrenyl)-maleimide, 1,1,3,3-tetramethoxypropane, and 2-vinyl pyridine were purchased from Aldrich (Milwaukee, WI). All other chemicals were purchased from Sigma (St. Louis, MO). HPLC grade reagents were used in GSH and MDA analysis.

All experiments were performed with Fisher 344 male rats weighing $75-100 \mathrm{~g}$. The animals were housed in stainless steel cages in a temperature-controlled room $\left(22^{\circ} \mathrm{C}\right)$ with a 12 -h light/ dark cycle. They were fed with standard rat chow (Purina rat chow). The animals were randomized into four groups. Group I $(n=11)$ served as the control and was given only standard rat chow and water for 6 weeks. Group II $(n=11)$ received $2000 \mathrm{ppm}$ lead acetate in its drinking water for 5 weeks and, during the 6th week, this group received water. Group III $(n=6)$ received 2000 ppm lead acetate in its drinking water for 5 weeks and, during the 6th week, these animals received 800 $\mathrm{mg} / \mathrm{kg} /$ day NAC dissolved in water. Group IV $(n=6)$ was treated like group III, except that it received $90 \mathrm{mg} / \mathrm{kg} /$ day succimer (instead of NAC) during the last week. At the end of the 6th week, after overnight fasting, the animals were anesthetized with metofane and blood samples were collected via intracardiac puncture using heparin as an anticoagulant. Plasma and the buffy coat were removed by centrifugation for $10 \mathrm{~min}$ at $3000 \mathrm{rpm}$. The RBCs were washed three times with an equal volume of cold saline. The samples were maintained at $-70^{\circ} \mathrm{C}$ for MDA assays (not longer than 7 days) and at $4^{\circ} \mathrm{C}$ for CAT assay (not longer than 4 days) (Aebi, 1984).

GSH determinations were performed using the HPLC methodology developed by Winters et al. (1995). HPLC determination of malondialdehyde (MDA) content was utilized as a marker of lipid peroxidation (Draper et al., 1993; Neal et al., 1997). Tissues $(0.140 \mathrm{~g} / \mathrm{ml})$ were homogenized in $100 \mathrm{mM}$ Tris buffer containing $10 \mathrm{mM}$ borate and $5 \mathrm{mM}$ serine with $1.00 \mathrm{mM}$ diethylenetriaminepentaacetic acid as described (Neal et al., 1997). Then, $0.250 \mathrm{ml}$ of homogenate was mixed with $0.650 \mathrm{ml}$ of $5 \%$ trichloroacetic acid (TCA) and $0.100 \mathrm{ml}$ of $500 \mathrm{ppm}$ butylated hydroxytoluene (BHT) in methanol. The sample was then heated in a boiling water bath for $30 \mathrm{~min}$. After cooling on ice, samples were centrifuged at $1000 \times g$ for $10 \mathrm{~min}$. The supernatant was mixed 1:1 with saturated thiobarbituric acid (TBA) and heated in a boiling water bath for $30 \mathrm{~min}$. After cooling on ice, $0.50 \mathrm{ml}$ of the samples were extracted with $1.00 \mathrm{ml}$ of $\mathrm{n}$-butanol and centrifuged to facilitate the separation of the two phases. The resulting organic layer was filtered through a $0.45-\mu \mathrm{m}$ acrodisc and then injected onto a reverse phase $250 \times 4.6 \mathrm{~mm} 3-\mu \mathrm{m} \mathrm{C}_{18}$ column. The mobile phase for this system was composed of $30 \%$ acetonitrile and $0.6 \%$ tetrahydrofuran in $5 \mathrm{mM}$ phosphate buffer $(\mathrm{pH}$ 7.0). The reaction complexes were eluted from the column isocratically at a flow rate of $0.70 \mathrm{ml} / \mathrm{min}$. 1,1,3,3-tetramethoxypropane was used to generate a standard curve and identification of the MDA-TBA adduct was made using comparison with retention times of the known standard. The data were expressed as nmol $\mathrm{MDA} / \mathrm{g} \mathrm{Hb}$. Catalase activity was determined spectrophotometrically (Aebi, 1984). The European standardized method was 
Table 1

Selective parameters indicative of oxidative stress in red blood cells from Fisher 344 rats

\begin{tabular}{lllcc}
\hline & Control & $\mathrm{Pb}$ & $\mathrm{Pb}+\mathrm{NAC}$ & $\mathrm{Pb}+\mathrm{SUC}$ \\
\hline GSH $(\mu \mathrm{mol} / \mathrm{g} \mathrm{Hb})$ & $24.5 \pm 1.2$ & $18.5 \pm 2.2^{* *}$ & $21.3 \pm 1.5^{\#}$ & $21.1 \pm 1.3^{\#}$ \\
MDA $(\mathrm{nmol} / \mathrm{g} \mathrm{Hb})$ & $39 \pm 7$ & $61 \pm 20^{*}$ & $36 \pm 6^{* * *}$ & $39 \pm 3^{* * *}$ \\
Catalase $(\mathrm{U} / \mathrm{g} \mathrm{Hb})$ & $100 \pm 30$ & $170 \pm 10^{* *}$ & $110 \pm 20^{* * *}$ & $130 \pm 10^{* * *}$ \\
G6PD $(\mathrm{U} / \mathrm{g} \mathrm{Hb})$ & $21.5 \pm 1.1$ & $26.2 \pm 1.5^{*}$ & $26.1 \pm 0.8$ & $22.5 \pm 2.3 *$ \\
ALAD $(\mathrm{U} / \mathrm{l})$ & $3.6 \pm 0.4$ & $0.9 \pm 0.3^{* *}$ & $0.7 \pm 0.1$ & $1.87 \pm 0.9$ \\
\hline
\end{tabular}

$\mathrm{Pb}$, lead; NAC, $N$-acetylcysteine; SUC, succimer; GSH, glutathione; G6PD, glucose-6-phosphate dehydrogenase; MDA, malondialdehyde; ALAD, aminolevulunic acid dehydratase; Hb, hemoglobin.

Values are averages of $3-11$ samples \pm S.D.

$* P<0.01$, compared to the corresponding value of control group.

** $P<0.001$, compared to the corresponding value of control group.

*** $P<0.01$, compared to the corresponding value of lead group.

\# $P<0.05$, compared to the corresponding value of lead group.

used to determine the whole blood ALAD activity (Berlin and Schaller, 1974). Determination of G6PD activity and the hemoglobin content were performed spectrophotometrically as detailed in Tietz (1986). The lead levels in whole blood were assayed by atomic absorption spectroscopy (Varian SpectrAA) by the CDC-certified analytical laboratory at the Springfield-Greene County Department of Public Health, Springfield, MO. The blood counts (hemoglobin, hematocrit, RBC, mean corpuscular volume, mean corpuscular hemoglobin, mean corpuscular hemoglobin concentration, and white blood cells) were measured by using Staks Counter by Coulter Corporation at Phelps County Regional Medical Center, Rolla, MO.

The Mann-Whitney $U$-test was used as a nonparametric test to analyze the significance of the differences between control and experimental groups.

\section{Results}

Table 1 shows selected parameters indicative of oxidative stress in RBCs from control and leadtreated animals. Significant decreases in GSH content and significant increases in MDA levels were noted in RBCs from lead-treated animals. Catalase and G6PD activities were significantly higher in lead-exposed animals when compared to controls. These results indicate an increase in lipid peroxidation and a decrease in intracellular reduced thiols in RBCs from lead exposed animals. In addition, the results with catalase and G6PD indicate an up regulation of enzymes associated with hydroperoxide metabolism and detoxification.

RBCs from lead exposed animals treated with NAC or succimer were shown to have significantly higher GSH levels and diminished MDA levels when compared to the lead group (Table 1). Catalase activity also returned to the control levels in lead exposed animals given NAC and succimer (Table 1). Succimer administration (but not NAC) resulted in decreased G6PD activity in RBCs from lead exposed animals (Table 1). These results show that selected parameters indicative of lead-induced oxidative stress in RBCs were reversed by administration of a thiol antioxidant or a metal chelator for 1 week following lead exposure.

Blood ALAD activity was significantly reduced $(75 \%)$ in lead-treated animals, when compared to control (Table 1). Inhibition of ALAD would be expected to result in $\delta$-aminolevulinic acid accumulation, which has been shown to be capable of autooxidizing to form reactive oxygen species such as hydroperoxides. These results are therefore consistent with the observations of increased lipid peroxidation, decreased GSH, and increases in enzymes associated with hydroperoxide metabolism. NAC treatment was incapable of restoring ALAD activity to control levels 1 week 
Table 2

Hematological parameters from whole blood analysis in control and experimental groups

\begin{tabular}{|c|c|c|c|c|}
\hline & Control & $\mathrm{Pb}$ & $\mathrm{Pb}+\mathrm{NAC}$ & $\mathrm{Pb}+\mathrm{SUC}$ \\
\hline HCT $(\%)$ & $48.8 \pm 0.55$ & $44.1 \pm 2.0^{*}$ & $44.4 \pm 0.5$ & $42.4 \pm 0.3$ \\
\hline $\operatorname{RBC}\left(10^{6} / \mu 1\right)$ & $8.98 \pm 0.07$ & $8.62 \pm 0.33^{*}$ & $8.89 \pm 0.1 * *$ & $8.56 \pm 0.03$ \\
\hline MCV (fl) & $54.2 \pm 0.3$ & $49.6 \pm 1.1^{*}$ & $49.7 \pm 0.4$ & $49.4 \pm 0.2$ \\
\hline $\mathrm{MCH}(\mathrm{pg})$ & $18.9 \pm 0.2$ & $17.7 \pm 0.2^{*}$ & $17.9 \pm 0.2$ & $17.9 \pm 0.1$ \\
\hline
\end{tabular}

HCT, hematocrit; Hb, hemoglobin; RBC, red blood cell; MCV, mean corpuscular volume; MCH, mean corpuscular hemoglobin; MCHC, mean corpuscular hemoglobin concentraion; WBC, white blood cell, Pb, lead; NAC, $N$-acetylcysteine; SUC, succimer. Values are averages of four samples \pm S.D.

$* P<0.05$, compared to the corresponding value of control group.

** $P=0.05$, compared to the corresponding value of lead group.

following lead exposure (Table 1). However, treatment with succimer did appear capable of partially restoring ALAD activity towards control levels, 1 week following lead exposure (Table 1), but this effect did not reach statistical significance.

Results presented in Table 2 show that lead exposure caused a significant reduction in hematocrit and hemoglobin content. Mean corpuscular volume (MCV) and mean corpuscular hemoglobin $(\mathrm{MCH})$ values were also significantly decreased, indicating microcytic hypochromic anemia. In contrast, white blood cell counts did not appear to be altered by exposure to lead. Treatment with NAC and succimer did not dramatically enhance the recovery of these hematological disturbances when given for 1 week following 5 weeks of exposure to lead.

Table 3

Blood lead levels from control and experimental groups

\begin{tabular}{lc}
\hline Groups & Blood lead levels $(\mu \mathrm{g} / \mathrm{dl})$ \\
\hline Control & $1.0 \pm 0.01$ \\
Lead & $34.8 \pm 4.1^{* *}$ \\
$\mathrm{~Pb}+$ NAC & $25.3 \pm 2.9^{*}$ \\
$\mathrm{~Pb}+$ SUC & $2.5 \pm 1.0^{* * *}$ \\
\hline
\end{tabular}

$\mathrm{Pb}$, lead; NAC, $N$-acetylcysteine; SUC, succimer.

All values represent mean \pm S.D.

Groups have three to five animals.

* Significantly different from lead group $(P<0.01)$.

** Significantly different from control group $(P<0.005)$.

$* * *$ Significantly different from lead group $(P<0.005)$.
Table 3 shows the blood lead levels. Animals exposed to lead for 5 weeks and then allowed to recover 1 week in the absence of continued lead exposure had blood lead levels of approximately $35 \mu \mathrm{g} / \mathrm{dl}$, which was reduced to $25 \mu \mathrm{g} / \mathrm{dl}$ by treatment with NAC during the 1-week recovery (Table 3). In contrast, the known chelating agent, succimer, lowered blood lead levels to $2.5 \mu \mathrm{g} / \mathrm{dl}$ when administered for 1 week following lead exposure. These results indicate that, following exposure, lead clearance from the blood stream was dramatically enhanced by succimer and only slightly enhanced by treatment with NAC. These results are consistent with the hypothesis that succimer's primary mode of action is through chelation and clearance of lead, while NAC's primary mode of action appears to be enhancement of thiol antioxidant capacity.

\section{Discussion}

The inability of known mechanisms of lead toxicity to explain some of the symptoms of lead poisoning (Waldron, 1966; Ratcliffe, 1981; Sugawara et al., 1991) has led to the investigation of alternative mechanisms. Recent studies have suggested lead-induced oxidative stress as a possible mechanism for some toxic effects of lead (Christie and Costa, 1984; Ercal et al., 1996; Neal et al., 1997). The hematological system has been proposed as being an important target organ for 
lead-induced toxicity. The effects of lead on this system are thought to result in decreased heme synthesis and anemia (Waldron, 1966; Ratcliffe, 1981). The present study was undertaken to investigate whether oxidative stress is involved in RBC damage caused by lead.

RBCs have a high affinity for lead and typically contain a majority of the lead found in the blood stream (De Silva, 1981; Leggett, 1993). The following factors make RBCs sensitive to oxidative damage: (1) RBCs are exposed to high concentrations of oxygen, (2) hemoglobin can be easily autoxidized, (3) RBC membrane components are vulnerable to lipid peroxidation, and (4) RBCs have limited capacity to repair their damaged components (Rice-Evans, 1990). Ribarov and Benov (1981) demonstrated in vitro that lead-induced hemolysis was associated with the peroxidation of RBC membranes and suggested that lead might exert this pro-oxidant effect by interacting with hemoglobin (Ribarov et al., 1981). Quinlan et al. (1988) showed that lead ions accelerate the peroxidation of RBC membrane lipids induced by hydrogen peroxide in the presence of azide. Furthermore, $\delta$-aminolevulinic acid (ALA), a heme precursor which accumulates during lead poisoning, has been shown to autooxidize resulting in the conversion of oxyhemoglobin to methemoglobin in a process that appears to involve the formation of superoxide and hydrogen peroxide (Monteiro et al., 1986). Increases in serum and RBC MDA content in workers exposed to lead has also been reported (Ito et al., 1985; Monteiro et al., 1985; Sugawara et al., 1991; Chiba et al., 1996). However it was not clear whether the results using RBCs from human subjects considered exposure to other environmental sources of oxidative stress. In addition, antioxidant administration was not included in these studies and, therefore, it is not known if lead-induced increases in parameters indicative of oxidative stress in vivo could be reversed by exogenous pharmacological manipulation of antioxidants.

The studies in the current report show that lead induced a decrease in RBC GSH content, which was partially reversed by a 1-week exposure to NAC or succimer following a 5-week exposure to lead acetate (Table 1). This data, in combination with the significant increase in RBC MDA content in lead-exposed animals (Table 1), suggests that lead stimulated lipid peroxidation in vivo resulting in the formation of aldehydic byproducts which, in turn, caused a decrease in reduced glutathione (GSH) content. This scenario seems plausible because aldehydic byproducts of lipid peroxidation have been suggested to decrease intracellular levels of $\mathrm{GSH}$, via the formation of aldehyde-GSH conjugates. In addition, lead has been suggested to bind sulfhydryl and glycine carboxylic groups of GSH (Christie and Costa, 1984) which could also deplete the intracellular levels of reduced GSH. Furthermore, it appears in vivo administration of either a thiol antioxidant (NAC) or a chelator is capable of inhibiting leadinduced increases in MDA levels as well as decreases in GSH content.

Further evidence for lead-induced oxidative stress arises from the increase in both RBC catalase and G6PD activity in lead-exposed animals (Table 1). G6PD, is the first committed step in the pentose phosphate pathway, supplying NADPH as a donor of reducing equivalents for glutathione reductase which mediates reduction of glutathione disulfide (GSSG) to GSH. The formation of GSSG would be expected to increase during the catalytic decomposition of hydroperoxides by glutathione peroxidase. G6PD activity would be expected to increase in response to increased fluxes of hydroperoxides to increase NADPH production. In fact, the increases in G6PD activity which were noted in RBCs of lead-treated rats in the present study confirm the results that Cocco et al. (1995) found in workers occupationally exposed to lead for 6 months. Regulation of the pentose phosphate pathway has not been studied extensively in lead poisoning, but the most important input into its regulation has been suggested to be the NADPH/NADP ${ }^{+}$ratio (Brigelius, 1986; Zubay, 1993). Therefore, increased utilization of NADPH for hydroperoxide metabolism during lead poisoning would appear to provide a plausible explanation for the stimulation of G6PD activity in RBCs from lead-treated rats.

Likewise increased catalase activity, noted in RBCs from lead-exposed animals, is consistent with increased fluxes of hydrogen peroxide during 
lead-induced oxidative stress. Catalase has been suggested to provide an important pathway for hydrogen peroxide $\left(\mathrm{H}_{2} \mathrm{O}_{2}\right)$ decomposition at higher steady state $\mathrm{H}_{2} \mathrm{O}_{2}$ concentrations in human RBCs, whereas glutathione peroxidase is believed to play a more important role in $\mathrm{H}_{2} \mathrm{O}_{2}$ decomposition under lower steady state levels of $\mathrm{H}_{2} \mathrm{O}_{2}$ (Cohen and Hochstein, 1963). In addition, previous workers have suggested that one of the functions of NADPH, which has been found to be bound to the catalase enzyme, may be to protect catalase against inactivation by $\mathrm{H}_{2} \mathrm{O}_{2}$ (Kirkman et al., 1987). If this is true, increasing catalase activity as a defense mechanism in RBCs from leadtreated rats might also contribute to an increased metabolic demand for NADPH, leading to the subsequent stimulation of G6PD activity.

The source of prooxidant production during lead-induced oxidative stress in RBCs is currently unknown. However, the present report as well as the work of others (Monteiro et al., 1986) suggests that lead-induced inhibition of ALAD could lead to accumulation of $\delta$-aminolevulinic acid. $\delta$-Aminolevulinic acid has been shown to undergo autooxidation resulting in the formation of superoxide and hydrogen peroxide (Monteiro et al., 1986). Furthermore, Quinlan et al. (1988) have shown that lead appears to exacerbate lipid peroxidation induced by $\mathrm{H}_{2} \mathrm{O}_{2}$ and iron. Since increased fluxes of superoxide and $\mathrm{H}_{2} \mathrm{O}_{2}$ are known to induce lipid peroxidation and oxidative stress, we hypothesize that lead-induced inhibition of ALAD leading to increased accumulation and autooxidation of $\delta$-aminolevulinic acid contributes to lead-induced oxidative stress in RBCs. In support of this hypothesis, we have previously shown that $\delta$-aminolevulinic acid induces oxidative damage in Chinese hamster ovary cells (Neal et al., 1997). Furthermore, our data also shows that chelation of lead using succimer (Table 3) appears to partially reverse the inhibition of ALAD seen 1 week following exposure to lead (Table 1).

The present study confirms the observation that lead exposure causes mild anemia along with other hematological disturbances, including considerable variability in the sizes of RBCs (anisocytosis) and irregular shapes in RBCs (poikilocy- tosis). These data, considered in light of the data suggesting that lead induces oxidative stress in RBCs, support the hypothesis that lead-induced oxidative stress could be, in part, responsible for the lead-induced toxicity to the hematological system. Although administration of NAC or succimer for 7 days following lead exposure did not result in significant improvement in parameters indicative of lead-induced injury to RBCs (Table 2), obvious reversal of selected parameters indicative of oxidative stress were noted (Table 1). In addition, the data in Table 3 show that NAC only slightly enhanced the clearance of lead from the blood stream and NACtreated animals still had blood lead levels indicative of lead intoxication. These results suggest that the beneficial effect of NAC on parameters of oxidative stress appear to be related solely to thiol antioxidant activity while the beneficial effects of succimer appear to result from chelation of lead and clearance from the blood stream. The fact that neither NAC nor succimer appeared to reverse the RBC injury parameters suggests that 1-week following lead exposure may not be enough time for the hematological system to recover from the effects of lead intoxication even in the presence of a thiol antioxidant or chelating agent. Further studies will be required to determine the effects of these treatments over a more prolonged period of time following exposure to lead. However, the speculation that NAC and succimer may be acting through different mechanisms (chelation versus bolstering thiol antioxidant capacity) suggests that combinations of these compounds may provide more benefit than either compound alone.

Studies indicate that succimer has advantages over conventional chelating agents, including a low incidence of side effects, ease of administration, and relative specificity for lead (Graziano et al., 1992; Aposhian et al., 1995). However, the antioxidant potential of succimer in the hematological system has not been rigorously examined. In a previous study (Ercal et al., 1996), we observed antioxidant effects of succimer in the brains and livers of lead-intoxicated mice. The current study shows that succimer has a visible protective effect on lead-induced oxidative stress 
in RBCs. However, since blood lead levels returned to control values in succimer-treated rats, it remains unclear if the observed effect of succimer can be attributed to chelation or the potential to act as a thiol antioxidant. It appears plausible that succimer could decrease parameters indicative of oxidative stress either by removing lead from the target cells and/or by directly scavenging ROS via its sulfhydryl groups. In fact other conventional chelating agents, such as Dpenicillamine, have also been shown to scavenge ROS in vitro (Benov et al., 1990). Further studies are needed to determine whether succimer exerts its beneficial effects solely through its ability to act as a metal chelator or whether part of its protective effects can result from its potential as a thiol antioxidant.

\section{Acknowledgements}

The authors are thankful to Gary Kellogg for his kind assistance in blood lead determinations and Dr De Lane Wiench for whole blood counts. Dr Nuran Erçal was supported by R15 ES08016 from the NIEHS. Hande Gürer was supported by The Scientific and Technical Research Council of Turkey (TUBITAK). Dr Douglas R. Spitz was supported by R01 HL51469 from NHLBI.

\section{References}

Aebi, H., 1984. Catalase in vitro. Methods Enzymol. 105, $121-126$.

Aposhian, H.V., Maiorino, R.M., Gonzalez-Ramirez, D., Zuniga-Charles, M., Xu, Z., Huelbut, K.M., Junco-Munoz, P., Dart, R.C., Aposhian, M.M., 1995. Mobilization of heavy metals by newer, therapeutically useful chelating agents. Toxicology 97, 23-38.

Aruoma, O.I., Halliwell, B., Hoey, B.M., Butler, J., 1989. The antioxidant action of $N$-acetylcysteine: its reaction with hydrogen peroxide, hydroxyl radical, superoxide, and hypochlorous acid. Free Radic. Biol. Med. 6, 593-597.

Banner, W. Jr., Koch, M., Capin, D.M., Hopf, S.B., Chang, S., Tong, T.G., 1986. Experimental chelation therapy in chromium, lead, and boron intoxication with $N$-acetylcysteine and other compounds. Toxicol. Appl. Pharmacol. 83, $142-147$.

Benov, L.C., Benchev, I.C., Monovich, O.H., 1990. Thiol antidotes effect on lipid peroxidation in mercury-poisoned rats. Chem.-Biol. Interact. 76, 321-332.
Berlin, A., Schaller, K.H., 1974. European standardized method for the determination of aminolevulinic acid dehydratase activity in blood. Zeitsch. Klin. Chem. Klin. Biochim. 12, 389-390.

Brigelius, R., 1986. Regulation of glucose-6-phospate dehydrogenase under oxidative stress. In: Rotilio G. (Ed.), Superoxide and Superoxide Dismutase in Chemistry, Biology and Medicine. Elsevier, Amsterdam, pp. 401-403.

Center for Disease Control, 1991. Preventing lead poisoning in young children. US Department of Health and Human Services, Center for Disease Control, Atlanta, GA.

Chiba, M., Shinohara, A., Matsushita, K., Watanabe, H., Inaba, Y., 1996. Indices of lead-exposure in blood and urine of lead-exposed workers and concentrations of major and trace elements and activities of SOD, GSH-Px and catalase in their blood. Tohoku J. Exp. Med. 178, 49-62.

Christie, N.T., Costa, M., 1984. In vitro assesment of the toxicity of metal compounds. IV. Disposition of metals in cells: interaction with membranes, glutathione, metallothionein, and DNA. Biol. Trace Elem. Res. 6, 139-158.

Cocco, P., Salis, S., Anni, M., Cocco, M.E., Flore, C., Ibba, A., 1995. Effects of short-term occupational exposure to lead on erythrocyte glucose-6-phosphate dehyrogenase activity and serum cholesterol. J. Appl. Toxicol. 15, 375-378.

Cohen, G., Hochstein, P., 1963. Glutathione peroxidase: the primary agent for the elimination of hydrogen peroxide in erythrocytes. Biochemistry 2, 1420-1428.

De Silva, P.E., 1981. Determination of lead in plasma and studies on its relationship to lead in erythrocytes. Br. J. Ind. Med. 38, 209-217.

Draper, H.H., Squires, E.J., Mahmoodi, H., Wu, J., Agarwal, S., Hadley, M.A., 1993. Comparative evaluation of thiobarbituric acid methods for the determination of malondialdehyde in biological materials. Free Radic. Biol. Med. 15, 353-363.

Ercal, N., Treeratphan, P., Hammond, T.C., Matthews, R.H., Granneman, N.H., Spitz, D.R., 1996. In vivo indices of oxidative stress in lead-exposed $\mathrm{C} 57 \mathrm{BL} / 6$ mice are reduced by treatment with meso-2,3-dimercaptosuccinic acid or $N$ acetylcysteine. Free Radic. Biol. Med. 21, 157-161.

Fukumoto, K., Karai, I., Horiguchi, S., 1983. Effect of lead on erythrocyte membranes. Br. J. Ind. Med. 40, 220-223.

Graziano, J.H., Lolacono, N.J., Moulton, T., Mitchell, M.E., Slavkovic, V., Zarate, C., 1992. Controlled study of meso2,3-dimercaptosuccinic acid for the management of childhood lead intoxication. J. Pediatr. 120, 133-139.

Haeger-Aronsen, B., Abdulla, M., Fristedt, B.I., 1971. Effect of lead on $\delta$-aminolevulinic acid dehydrase activity in red blood cells. Arch. Environ. Health 23, 440-445.

Hasan, J., Vihko, V., Hernberg, S., 1971. Deficient red cell membrane $\left(\mathrm{Na}^{+}-\mathrm{K}^{+}\right)$-ATPase in lead poisoning. Arch. Environ. Health 14, 313-318.

Ito, Y., Niiya, Y., Kurita, H., Shima, S., Sarai, S., 1985. Serum lipid peroxide level and blood superoxide dismutase activity in workers with occupational exposure to lead. Int. Arch. Occup. Environ. Health 56, 119-127. 
Kirkman, N.H., Galiano, S., Gaetani, G.F., 1987. The function of catalase-bound NADPH. J. Biol. Chem. 262, 660666.

Lawton, L.J., Donaldson, W.E., 1991. Lead-induced tissue fatty acid alterations and lipid peroxidation. Biol. Trace Elem. Res. 28, 83-97.

Leggett, R.W., 1993. An age-specific kinetic model of lead metabolism in humans. Environ. Health Perspect. 101, 598-616.

Moldeus, P., Cotgreave, I.A., Berggren, M., 1986. Lung protection by a thiol-containing antioxidant: $N$-acetylcysteine. Respiration 50, 31-42.

Monteiro, H.P., Abdalla, D.S.P., Arcuri, A.S., Bechara, E.J.H., 1985. Oxygen toxicity related to exposure to lead. Clin. Chem. 31, 1673-1676.

Monteiro, H.P., Abdalla, D.S.P., Faljoni-Alario, A., Bechara, E.J.H., 1986. Generation of active oxygen species during coupled autoxidation of oxyhemoglobin and $\delta$ aminolevulinic acid. Biochim. Biophys. Acta 881, 100-106.

Neal, R., Yang, P., Fiechtl, J., Yildiz, D., Gurer, H., Ercal, N., 1997. Pro-oxidant effects of $\delta$-aminolevulinic acid on Chinese hamster ovary cells. Toxicol. Lett. 91, 169-178.

Prescott, L.F., Park, J., Ballantyne, A., Adriaenssens, P., Proudfoot, A.T., 1977. Treatment of paracetamol (acetaminophen) poisoning with $N$-acetylcysteine. Lancet ii, 432-434.

Quinlan, G.J., Halliwell, B., Moorhouse, C.P., Gutteridge, J.M.C., 1988. Action of lead (II) and aluminium (III) ions on iron-stimulated lipid peroxidation in liposomes, erythrocytes and rat liver microsomal fractions. Biochim. Biophys. Acta 962, 196-200.

Ratcliffe, J.M., 1981. Lead in Man and The Environment. Ellis Horwood, Chichester, pp. 34-47.
Ribarov, S.R., Benov, L.C., 1981. Relationship between the hemolytic action of heavy metals and lipid peroxidation. Biochim. Biophys. Acta 640, 721-726.

Ribarov, S.R., Benov, L.C., Benchev, I.C., 1981. The effect of lead on hemoglobin-catalyzed lipid peroxidation. Biochim. Biophys. Acta 664, 453-459.

Rice-Evans, C., 1990. Iron-mediated oxidative stress and erythrocytes. In: Harris, J.R. (Ed.), Blood Cell Biochemistry, vol. 1. Plenum Press, New York. pp. 429-453.

Sandhir, R., Gill, K.D., 1995. Effect of lead on lipid peroxidation in livers of rats. Biol. Trace Elem. Res. 48, 91-97.

Sandhir, R., Julka, D., Gill, K.D., 1994. Lipoperoxidative damage on lead exposure in rat brain and its implications on membrane bound enzymes. Pharmacol. Toxicol. 74, 66-71.

Sugawara, E., Nakamura, K., Miyake, T., Fukumura, A., Seki, Y., 1991. Lipid peroxidation and concentration of glutathione in erythrocytes from workers exposed to lead. Br. J. Ind. Med. 48, 239-242.

Tietz, N.W., 1986. Textbook of Clinical Chemistry. W.B. Saunders, Philadelphia.

Waldron, H.A., 1966. The anemia of lead poisoning: a review. Br. J. Ind. Med. 23, 83-100.

Winters, R.A., Zukowski, J., Ercal, N., Matthews, R.H., Spitz, D.R., 1995. Analysis of glutathione, glutathione disulfide, cysteine, homocysteine, and other biological thiols by highperformance liquid chromatography following derivatization by $N$-(1-pyrenyl)maleimide. Anal. Biochem. 227, 14-21.

Ziment, I., 1986. Acetylcysteine: a drug with an interesting past and a fascinating future. Respiration 50 (Suppl. 1), 5-12.

Zubay, C., 1993. Biochemistry. Vol. II: Catabolism and Biosynthesis. Brown, Dubuque, pp. 351. 